AsClepIO. Revista de Historia de la Medicina y de la Ciencia

69 (2), julio-diciembre 2017, p192

ISSN-L:0210-4466

http://dx.doi.org/10.3989/asclepio.2017.15

DOSSIER: ESCENARIOS DE LAS PRÁCTICAS PSIQUIÁTRICAS EN AMÉRICA LATINA (SIGLOS XIX Y XX) /

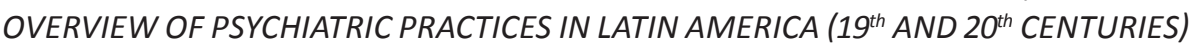

\title{
DE LA CASA DE ORATES AL OPEN DOOR: EL PAISAJE EN EL PROYECTO ASILAR CHILENO, 1852-1928
}

\author{
María José Correa Gómez \\ Departamento de Humanidades, Universidad Andrés Bello. \\ maria.correa@unab.cl \\ ORCID iD: http://orcid.org/0000-0002- 4252-8538
}

Recibido: 07 abril 2017; Aceptado: 06 junio 2017.

Cómo citar este artículo/Citation: Correa Gómez, María José (2017), “De la Casa de Orates al Open Door: el paisaje en el proyecto asilar chileno, 1852-1928", Asclepio 69 (2): p192. doi: http://dx.doi.org/10.3989/asclepio.2017.15

RESUMEN: En un contexto de desarrollo de la anatomía patológica y de la irrupción de la mirada científica sobre el cuerpo de los enfermos mentales, los asilos de locos subrayaron la importancia del espacio, real o simulado, como recurso curativo. Las Casas de Orates se armaron a partir de una propuesta que en su diseño, emplazamiento y realización representó los principios del alienismo, ajustados a la realidad chilena. Este proceso impulsó un escenario asilar específico, con paisajes interiores y exteriores, que caracterizaron al alienismo local y a su promesa de tratar la locura. Este artículo estudia los asilos proyectados o levantados en Chile desde la Casa de Locos (1852) hasta el Open Door Nacional (1928), dando cuenta de los procesos de apropiación de un modelo internacional desde las particularidades del paisaje local asilar.

PALABRAS CLAVE: Casa de Orates; Tratamiento Moral; Espacio Terapéutico; Chile; Siglos XIX y XX.

\section{FROM THE MADHOUSE TO THE OPEN DOOR: THE LANDSCAPE IN THE CHILEAN ASYLUM PROJECT, 1852-1928}

ABSTRACT: In a period shaped by the development of anatomical pathology and by the entrance of the scientific gaze over the body of the insane, the mental asylum underscored the key importance of the space, real or simulate, as a therapeutic tool. Madhouses were influenced by a proposal that followed in terms of design, location and implementation, the principles of alienism, adjusted to the Chilean setting. This process contributed to develop a specific asylum space, with internal and external landscapes, which characterized local alienism and its promise to treat madness. This article studies the Chilean asylums -planned or built- from the Madhouse (1852) to the National Open Door (1928), in order to show the process of appropriation of an international therapeutic model from the peculiarities of the institutional landscape.

KEY WORDS: Madhouse; Moral Treatment; Therapeutic Landscape; Chile; XIX and XX Centuries. 
La creación de la primera Casa de Orates de Chile en 1852 representó un momento fundacional en la conceptualización de la locura. Su instalación en Santiago implicó la apropiación de un modelo terapéutico que se situó como epicentro e ideal del desarrollo alienista de las siguientes décadas. La institución ofreció, con el paso del tiempo, un espacio curativo inédito, y representó un lugar de construcción de identidad, saber, autoridad y sedición, que la proyectó durante el siglo XX como un sitio protagónico del estudio y administración de la locura, con sus matices y vaivenes. Locos, orates, dementes y furiosos, entre los varios calificativos utilizados para nombrar a quienes parecían haber perdido la razón, se encontraron con un espacio de internación que determinó su capacidad, su condición y su accionar. Médicos y estudiantes de medicina se vincularon con un sitio formativo y de definición profesional, que les permitió desarrollar la nueva rama del alienismo. Autoridades y vecinos reconocieron un espacio que separó la razón de la insania y que ofreció contención a la locura furiosa y curación a la afección mental. Y la locura, en tanto problema y pregunta, se identificó con un territorio de creación de conocimiento científico y de formalizaron de categorías patológicas centrales para el posicionamiento del alienismo en la sociedad.

La propuesta europea de aislar a los locos para limitar las consecuencias de sus acciones y de contribuir a su cuidado, cruzó al continente americano influyendo en las instituciones de asistencia y escuelas de medicina. Como resultado se construyeron en la región más de una decena de Casas de Orates, surgieron los llamados alienistas y se crearon las primeras cátedras universitarias de enfermedades mentales. Estos elementos actuarían como signos protagónicos del proceso de medicalización de la locura en Chile, y de su conceptualización como una enfermedad capaz de ser curada, o al menos, aminorada, a través de distintos recursos, entre ellos, la internación de los enfermos en recintos de salud regidos por los preceptos del tratamiento moral. La implementación de este último estaría acompañada por la entrada de idearios escoceses y franceses, asociados a figuras como William Cullen (1719-1790), Philippe Pinel (1745-1826) y Jean Esquirol (1772-1840), arribados al país por diversos canales, como la formación que recibió el fundador de la primera Escuela de Medicina del Chile republicano, William Blest (18001884) en la University of Edinburgh, o la influencia francesa absorbida por los forjadores del alienismo nacional, José Ramón Elguero (1821-1897), Carlos Sazié (1852-1921) y Augusto Orrego Luco (1849-1933), entre otros, en la Universidad de Chile y en el extranjero.
La Casa de Orates representó un sitio de construcción de identidad y de poder, al mismo tiempo que se constituyó como marco material del alienismo y como espacio de ensayo de sus recursos curativos. Encarnó las potencialidades y al mismo tiempo las dificultades de la medicina chilena y de sus instituciones de beneficencia para administrar la salud mental de la población. Esta doble caracterización no resulta contradictoria, sino más bien refleja las particularidades de los procesos de apropiación nacional de modelos terapéuticos europeos y las dinámicas que acompañaron la construcción de un alienismo local, con todas sus complejidades y tonos. Es esta ambivalencia la que este artículo se interesa en recorrer, desde los espacios y paisajes asilares propuestos, diseñados o implementados entre 1852 y 1928. De esta forma, y siguiendo la noción de landscape (Mitchell, 2002), busca revisar el proyecto y sus características, entendiendo a sus espacios como recursos activos y dinámicos, gestadores de identidades y modificadores activos del modelo representado; como soportes del patrón asilar, que sustentaron y unieron sus paisajes plurales, al mismo tiempo que contribuyeron a dibujar una escena más amplia asociada al programa higiénico de la nación (Cosgrove, 2002).

Este artículo revisa y recorre los primeros espacios manicomiales de Chile: la Casa de Orates de Santiago (1852 y 1856), la Casa de Orates de Providencia (1894), el Manicomio de Concepción (1895) y el Opendoor del Peral (1928). Explora los espacios institucionales que acompañaron el tránsito de la Casa de Orates al Open Door, revisando su materialidad difusa, quimérica, precaria y contradictoria, forjadora de paisajes con continuidad, reales e imaginados, deseados y cuestionados. El surgimiento de sitios de locura y su apropiación por parte de una comunidad de personas y saberes, definió el encierro psiquiátrico. En este sentido, y siguiendo una lectura foucaultiana del espacio que permite ver a la Casa no como un sitio vacío e impuesto, sino como un espacio heterotópico, definido y ordenado desde las diversas relaciones en ella desarrolladas, vinculadas a su vez con distintos niveles del hacer, asociados a deseos, rebeliones y realizaciones de la comunidad que interactúa con ella (Foucault y Miskowiec, 1986), este estudio propone un recorrido por los espacios asilares forjados en el país entre 1852 y 1928 y un análisis preliminar de sus formas y usos. Revisa a través de fuentes diversas ${ }^{1}$, los cambios del paisaje asilar desde la Casa al Manicomio, y desde la propuesta de tratamiento moral al de puertas abiertas, en una contribución general, pero necesaria (Sacristán, 2009). En esta aproximación busca relacionar 
el ámbito material con el terapéutico y contribuir a una discusión sobre el valor del paisaje y del espacio en el proyecto asilar (Hickman, 2005; Edginton, 2003; Mackinnon, 2009).

La Casa de Orates determina nuevos "contra-espacios", espacialidades imaginarias que se sustentan en espacios reales y que revelan la pluralidad y heterogeneidad, pero al mismo tiempo la unidad, del lugar de la locura asilada. Este énfasis busca ir más allá de las tesis totalizantes heredadas de los sesenta y setenta respecto a los asilos médicos como dispositivos eficaces al servicio del proyecto liberal (Goffman, 1961), con el objeto de revisar otras agencias y contextos que colaboraron en la implementación local del ideal médico reformista. El manicomio fue, como propone Valeria Pita para el caso bonaerense, una construcción histórica compleja que respondió a conflictos, imaginarios, proyectos políticos, representaciones colectivas, en ocasiones "distantes de lo que hoy en día reconocemos, aceptamos o rechazamos" (Pita, 2012, p. 14). En base a este reconocimiento, interesa visitar sus espacios y pensar sus paisajes, para analizar el molde particular del modelo de tratamiento moral.

\section{EL MODELO ASILAR DECIMONÓNICO EN LA CASA DE ORATES DE LOS OLIVOS}

El plano del arquitecto Pedro Dejean identificaba en 1856 una pequeña construcción llamada Loquería² ${ }^{\text {. Al }}$ poniente de la capital y separada de ella por chacras y viñedos, la Casa de Orates de Nuestra Señora de los Ángeles, fundada en agosto de 1852, se elevaba como uno de los primeros esfuerzos institucionales por tratar médicamente la locura. A esta iniciativa, se sumaría en 1856 una segunda institución - la Casa de Orates de los Olivos- que, ubicada en la periferia nororiente de Santiago, reemplazaría a la Casa de Yungay (Osorio, 2016, p. 391).

La creación de este establecimiento se inspiró en los nuevos paradigmas decimonónicos sobre la locura y su administración, generosamente abordados por la historiografía internacional (Grob, 1973, Rothman, 1971 y Goldberg, 2001). Herederos de ideas preexistentes sobre la integración del cuerpo y la mente, y receptores del proceso de reducción de la locura a una condición cerebral, estos arquetipos subrayaron la importancia de lo corporal y lo síquico en la terapéutica mental y permitieron el desarrollo del llamado tratamiento moral, un modelo curativo que buscó sanar la locura a través del aislamiento y la disciplina y que se implementó primero en los asilos de locos europeos del siglo XVIII y principalmente del XIX (Huer- tas, 2008). Estos establecimientos sirvieron de modelo para su desarrollo en América Latina a mediados del siglo XIX, en una coincidencia temporal regional que reflejó la apropiación local del marco ideológico, en un sentido que evidentemente se distanció de las condiciones de emergencia y producción europea, y que entregó características disímiles propias de los contextos locales.

La Casa de los Olivos fue presentada como una necesidad social, acorde a todo país civilizado ${ }^{3}$. Metaforizó los cambios enfrentados por la enajenación mental, que dejaba de ser comprendida como un estado de endemoniamiento, para pasar a constituirse como "una enfermedad como cualquiera", que debía inspirar "interes i compasión". Este giro introducía el tratamiento racional del asilo e intentaba abandonar, en palabras de Manuel Beca, el abordaje inhumano que llevaba a los locos a enfrentarse con suplicios que iban "desde el cadalzo hasta la hogera", o al olvido silencioso que los hacía vagar precariamente por el país ${ }^{4}$. El tratamiento moral, considerado por José Ramón Elguero -primer alienista del asilo- como el único capaz de ofrecer resultados eficaces, se sostenía en la separación del enfermo de su familia y de las relaciones y hábitos que incidían en su estado patológico ${ }^{5}$. Sostenido sobre el efecto benéfico de alejar al enfermo de la vida citadina, que comenzaba a ser vista como epicentro patológico y como sitio malsano, comprendía el acceso a un nuevo espacio terapéutico en el que confluían una variedad de recursos terapéuticos adicionales que complementaban y al mismo tiempo definían la aislación (Correa, 2009 y 2013).

El tratamiento moral sostuvo el desarrollo de la institución, gracias a una promesa curativa que ofrecía reducir las desviaciones de la mente por medio de la internalización de las normas del régimen asilar. Este sistema se presentaba como capaz de modificar voluntades, afectos y sensibilidades desviadas, y se mostraba como parte de un giro epistemológico que caracterizaba a la locura como una afección de la voluntad y la sensibilidad, es decir, de las facultades del hacer y sentir. Bajo estos lineamientos, la propuesta del asilo brindaba un espacio de contención y salud diferenciado, que inauguraba mecanismos para movilizar y transformar estas facultades, entre los que se consideraba el espacio, por ser determinante en la aislación y por vigilar los factores que impresionaban $y$, por tanto, afectaban los sentidos de los internos.

La Casa se inspiró en el aislamiento y el retiro, y desde esa perspectiva dibujó su paisaje. Correspondió a una institución demarcada físicamente, circundada 
por viñas y chacras, ubicada en la ciudad, pero separada de ella simbólicamente, sea por hitos, como el río Mapocho, que diferenciaba la urbe ilustrada, de los arrabales y la ruralidad, o por su posicionamiento en los márgenes urbanos (Castillo, 2014). La Casa se presentó, en dicha periferia, como una chispa de modernidad, que introdujo a los extramuros elementos propios del desarrollo científico e intelectual citadino, y que con el tiempo contribuyó, junto a otros recintos médicos y hospitalarios, a la expansión del suelo urbano. Su edificio, a cargo del arquitecto Fermín Vivaceta (1829-1890), fue inaugurado pese a estar inconcluso, acogiendo inicialmente a un número menor que los 270 internos proyectados. De adobe y de madera, con piso de ladrillo, la casa cambió su materialidad con el paso del tiempo. Su suelo se reemplazaría por un entablado de madera, se cambiarían los pesados candados de las puertas por chapas y se abrirían nuevos arcos y ventanas en reemplazo de los antiguos tragaluces. Contaba con alrededor de diez patios, cinco por lado y dos fuentes de agua, además de acequias que comunicaban los patios y atravesaban el asilo las que serían substituidas a fines del siglo por cañerías para evitar anegaciones y problemas de higiene ${ }^{6}$.

Durante sus primeros años la Casa contó con pocos internos. Si bien en Yungay la media anual no superó las 30 personas, en los Olivos su número se elevó a 367 en 1870 y 634 en 1879, expandiéndose a 1773 a fin de siglo y a 2100 hacia 1922, para un país que había visto crecer sostenidamente su población, de 1.439.120 a 3.753.799 habitantes entre 1854 y $1922^{7}$. Gran parte de los insanos fueron identificados como maníacos, dementes o fatuos. Con el tiempo dichas clasificaciones generales disminuyeron, frente a una carta de afecciones más diversa, que amplió la paleta diagnóstica. Al mismo tiempo, comenzaron a predominar ciertas patologías, como el delirio alcohólico, que si bien en 1896 solo aludía a 38 internos, representaba el mayor problema entre los que acudían a la institución, con un total de 117 casos $^{8}$.

El aumento y la diversificación de la población determinaron cambios en la infraestructura. Se crearon los pensionados, secciones pagadas que con sus propios guardianes, cocinera y espacios privados reproducían las jerarquías sociales del exterior. Se levantaron recintos especiales para dar cabida a ciertos tipos de enfermos y a sus necesidades terapéuticas, particularmente a alcohólicos bajo el Asilo de Temperancia (Fernández, 2009). La ley de alcoholes empujó estos cambios, y hacia 1925 oficializó el cuidado de los alcohólicos crónicos y reincidentes, lo que se había implementado de facto hacía ya un tiempo ${ }^{9}$. También empujaron estos cambios las grandes transformaciones que experimentó el alienismo entre mediados del siglo XIX e inicios del XX, bajo los presupuestos de las ideas de la anatomía patológica, la degeneración y el impulso de la higiene social (Araya, 2015, Sánchez, 2014 y Ruperthuz y Sánchez, 2015). De esta manera el establecimiento cambió su espacio y sus paisajes de acuerdo a los presupuestos médicos que ampliaban el registro etiológico y a las soluciones asociadas a ellos, promoviendo el ingreso de nuevos tipos de enfermos y de terapias. Entre la oferta terapéutica del proyecto moral se encontraban, por una parte, como indicaban los mismos médicos del establecimiento, los "medios higiénicos" o acervos morales, conceptualizados como constitutivos de la terapia asilar, como el aislamiento, el entretenimiento, la ocupación, el trabajo, la lectura, la disciplina, la reglamentación ordenada y el buen empleo del tiempo ${ }^{10}$. Por otra parte, se ubicaban los "medios terapéuticos" que correspondían a la aplicación de medicamentos y drogas, y a los agentes "psico-físicos" -como el agua, la electricidad y el hipnotismo- propuestos por la medicina.

Los "medios higiénicos", comandados por el retiro y el encierro, fueron centrales a la propuesta de la Casa y entre sus beneficios se encontraba su capacidad de generar quietud, alejar al enfermo de las causas de exitación, protegerlo de reacciones nuevas y desarrollar elementos eficaces para su salud mental ${ }^{11}$. Estos buscaron activar los recursos morales del sistema asilar y determinaron marcadamente las características materiales y espaciales del asilo. La internación del enfermo definió a la Casa como un espacio cerrado. Muros y puertas separaban el mundo exterior del interior, junto a normas y procesos estipulados por administradores, jueces y médicos. El aislamiento debía ser asegurado por condiciones materiales, vigiladas por estatutos que fijaban la vinculación y conexión del espacio interno con el externo. A la Casa de Orates se entraba y salía siguiendo los reglamentos de 1856, 1883 y 1927, que regían la aislación y ordenaban el asilo para los interdictos, furiososos o que causasen escándalo, presos enjuiciados, indigentes, o dementes remitidos por un curador, autoridad o pariente, y permitían la salida a quienes hubiesen sido reconocidos por el médico como curados, o en caso de no estar sanados, fueran retirados por los familiares o por quiénes los habían internado ${ }^{12}$.

El ideario católico, nutriente terapéutico del proyecto asilar, se escenificó en la capilla que se ubicaba al centro del asilo, rodeada, como muestran los ma- 
pas $^{13}$, de jardines y corredores. Con grandes ventanas por las cuales los internos del credo católico podían "ver" las misas celebradas los domingos y festivos, recibía a los favorecedores del asilo, y a todos quienes de una u otra forma se vinculaban con el establecimiento. De esta forma la capilla ofrecía un espacio donde la razón se intersectaba con la insania bajo la vigilancia de la religión. Este diálogo fue respaldado por las normativas internas del establecimiento, las que obligaron la presencia de un capellán, cuya silueta se mezclaría con las de las religiosas que a fines del siglo administrarían la institución. El reglamento de 1883 estableció, que la Casa debía contar con un sacerdote para la misa y la administración del sacramento de la penitencia a "los enfermos que lo soliciten i el estado de su cerebro lo permita"14. A esta presencia se sumó el interés de las autoridades de poner el asilo en manos de religiosas. Desde el inicio se discutió sobre la conveniencia de traer a congregaciones como las Hermanas de la Caridad y las Monjas de la Providencia. Finalmente, en 1895, arribaron las religiosas de San José de Cluny, contratadas en París por el gobierno para hacerse cargo de los servicios generales de administración y también de la sección de mujeres. Esta presencia se relacionaba con una política implementada por el gobierno respecto a los sitios de salud, que veía en el apoyo católico un empuje para el desarrollo de la nueva medicina hospitalaria de inicios del siglo $\mathrm{XX}$, como engranaje del paso de la beneficencia a la salud pública (Ponce de León, 2011). La presencia de la religión, patrón compartido por otros estableciamientos, como los nuevos hospitales que se construyeron a fines del XIX que entregaron varias de las tareas centrales a congregaciones católicas, se visualizó en los diferentes signos del paisaje asilar, como la capiIla, los crucifijos, las figuras de las monjas, los cantos religiosos, entre otros.

El cruce entre medicina y religión explica también el recorrido administrativo de la Casa durante el siglo XIX. Como espacio de beneficencia pública, fue administrada hasta 1891 por una junta integrada por representantes de la elite y de la Iglesia, pasando luego a depender de la Junta de Beneficencia de la Capital. Como institución sostenida por la caridad mostró problemas para alcanzar los proyectos que se presupuestaban y para desarrollar su potencial. Por una parte, la tensa relación entre los médicos y la Junta Directiva, y que en ocasiones llevó a la destitución de algún especialista, obstaculizó el trabajo en conjunto de los hombres de ciencia y los de caridad (Camus, 1993). Estos últimos parecieron no comprender del todo la finali- dad curativa del sistema asilar, reduciendo el accionar del médico a la aplicación de medicinas, sin permitirles participar de la toma de decisiones respecto del funcionamiento de la institución, y por tanto, de las condiciones de aplicación del tratamiento moral. Por otra, los recursos estatales fueron reducidos, apoyándose en la caridad de las elites y la limosna pública, graficada en las dos alcancías ubicadas en la entrada principal para recibir donaciones.

Entre los medios higiénicos promulgados por el asilo, destacó el uso del tiempo libre. Si bien las críticas respecto a la ociosidad de los internos fueron abundantes, se diseñaron varios proyectos para fomentar su movimiento, los que incidieron a su vez sobre el espacio y sus usos. Citando los postulados de alienistas europeos y aludiendo a los logros alcanzados en los asilos extranjeros, Sazié y sus contemporáneos, valoraron el quehacer productivo, útil además a la economía del establecimiento. El trabajo activaba el cuerpo, haciendo descansar el cerebro de las ideas fijas, las divagaciones y la fantasía, ayudaba al "reposo del espíritu por el alejamiento de las preocupaciones enfermizas del loco", mantenía la salud física y producía un "equilibrio de las fuerzas utilizándolas"15. Esta atención se tradujo en la creación de propuestas laborales definidas por el género y anudadas a las necesidades del asilo. Para los hombres se impulsaron talleres de zapatería, para la fabricación del calzado de los internos, y de carpintería, para el arreglo del espacio asilar. Luego se sumaron otros de herraje, hojalatería y gasfitería, junto a labores agrícolas $^{16}$. Las mujeres accedieron a talleres diferenciados, menos provistos espacialmente. Cosían al aire libre los vestidos de internas indigentes, y algunas trabajaban en la lavandería. Pese al impulso y quizás como consecuencia de la demora en su implementación, la participación de los internos en los talleres fue baja durante las primeras cinco décadas de funcionamiento de la institución, incrementándose en las primeras décadas del siglo XX (Torres, 2014). Con el tiempo, el espacio psiquiátrico continuó ofreciendo talleres, orientados a apoyar la economía interna del establecimiento, con un alcance limitado y con una trayectoria fragmentada. La historiografía se ha interesado en este propósito, en tanto signo político y moral del llamado dispositivo asilar (Leyton, 2005), sin embargo, más allá de la retórica visual y promocional, la institución continuó operando en base a carencias y limitaciones que no permitieron transformar la laborterapia en un modelo terapéutico eficiente y efectivo al proposito terapéutico y económico del asilo, y posteriormente del manicomio. 
No solo el trabajo apelaba al control de la mente y su divagación, sinto también el entretenimiento como base sustantiva del tratamiento moral (MacKinnon, 2009). Sazié destacaría las actividades recreativas ofrecidas en los asilos extranjeros, bastante más nutridas que las brindadas por el asilo de Santiago, restringidas a la práctica de bolas y palitroques, mientras que el alienista Germán Greve enseñaría que todos los "asilos modernos", contaban con "juegos agradables y recreativos, palitroques, billares", como de "salones de espectáculos, bailes y soirées musicales"17. La reducida oferta del asilo de Santiago se amplió tras la construcción de uno de sus espacios más emblemáticos, el teatro inaugurado en 1897, siguiendo indicaciones que se remontaban al menos hacia 1875, cuando Benham sugirió construir "un grande espacio o salón cubierto" para comedor y para diversiones, como conciertos y bailes ${ }^{18}$. El Teatro o Salón Grez se edificó como muchas otras iniciativas gracias a un acto filantrópico, bajo la administración de Pedro Montt, quién sería presidente del país entre 1906 y 1910, periodo en el cual se realizaron importantes cambios en la Casa en un afán de reposicionar y cumplir sus espectativas ${ }^{19}$. Acompañaron la definición de un espacio teatral, los esfuerzos de constitución y habilitación de espacios comunes para los internos asociados al canto, al rezo, a la comida, a la lectura y a la actuación, acomodados a su vez en jerarquías patológicas y de comportamiento. Estos lugares ampliaron la circulación, favorecieron la interacción entre hombres y mujeres, y sirvieron como puente para la interacción con la comunidad en general, más allá de la asilar.

Junto a las acciones directas que activaban la pedagogía moral, se ofrecieron tratamientos más específicos, que podemos asociar a "los medios terapeuticos" identificados por los facultativos del establecimiento. Estos si bien se modificaron con el paso del tiempo, apelaron al uso de medicinas - preparados de botica y luego específicos- y la aplicación de sangría, así también de electricidad e hidroterapia, entre otros. Estos sistemas, determinaron el espacio e impulsaron la creación de gabinetes y recintos dirigidos al uso de la nueva tecnología en circulación. En el transcurso del siglo se rehicieron los baños de duchas y de lluvia, y siguiendo los principios de la hidroterapia se le dio importancia a los baños tibios de tina. A fines del siglo XIX se construyó un gabinete de electricidad, con aparatos nuevos, costosos y eficaces, que constituían, según los médicos, "la mejor instalación en su jénero" en los establecimientos de la beneficencia pública ${ }^{20}$. La importancia de la anatomopatología y la vinculación de lesiones orgánicas con condiciones mentales incentivaron el estudio del cuerpo. Como consecuencia, la
Casa se dotó de instrumentos quirúrgicos para operaciones y autopsias, y de un laboratorio de microscopía, microfotografía y fotografía, y un número cada vez más elevado de médicos realizando investigación.

En 1900, el doctor Carlos Ugarte, encargado del laboratorio, informaba con satisfacción y confianza al administrador, en un intento quizás de ampliar los recursos destinados a su área, que los numerosos estudios realizados en el laboratorio habían contribuido significativamente a esclarecer los diagnósticos de los pacientes. Los exámenes de orina en melancólicos y maníacos mostraban datos interesantes. En el caso de la melancolía identificaba un aumento considerable de ciertas sustancias en el intestino, interpretados como "verdaderos venenos del sistema nervioso" 21 . Las autopsias y análisis histológicos establecían causas de muerte y permitían cruces con el historial médico de los internos. El reconocimiento del valor del estudio del cuerpo llevó a que el asilo se convirtiera en el único establecimiento que siguiendo a las clínicas europeas, contaba hacia 190 con 900 autopsias protocolarizadas y más de 100 piezas anatómicas ${ }^{22}$. A esto se sumaban secciones de bacteriología para la realización de examenes de espectoración, y de química para la ejecución de examenes de orina, jugo gástricos, líquidos patológicos y leche $u$ alimentación. Junto con apoyar la estadística, estas instancias mostraban el potencial productivo del establecimiento, aportando algunas conclusiones preliminares, como las de Carlos Ugarte sobre algunos procesos internos relacionados con ciertos estados mentales. En su interés por dialogar con las investigaciones europeas respecto al origen y desarrollo de las enfermedades mentales, comprobaba "los resultados obtenidos por algunos autores como Ballet, Regis i Chevallier" respecto a una disminución de la toxicidad urinaria en la manía y un aumento en la melancolía ${ }^{23}$.

Durante la segunda mitad del XIX la implementación y el perfeccionamiento del tratamiento moral se incrustó a un determinado paisaje asilar que desde sus usos y formatos determinó al proyecto chileno. Si bien adolecía de múltiples problemas y carencias, este se organizó y se presentó desde los espacios conquistados, más que desde las posibilidades reales de sanación ofrecidas. El siglo cerraba con aires de reforma, que llevarían a la búsqueda de soluciones y la implementación de nuevos espacios, que renovarían la propuesta, pero permitirían interesantes traspasos. Como resultado, la última década del siglo, se caracterizó por un dinamismo que acogió debates y proyectos, y que impulsó futuros establecimientos como los Manicomios de la Providencia y de Concepción, y el Open Door del Peral. 
LOS NUEVOS MANICOMIOS, LA NECESIDAD DE REFORMA Y EL VALOR DEL PAISAJE

Para fines del siglo XIX el establecimiento asilar de Santiago se hacía insuficiente para cubrir las necesidades nacionales. Pese a que el reconocimiento de sus problemas se remontaba a décadas atrás, los cambios en sus espacios y en sus presupuestos terapéuticos no habían logrado aplacar las dificultades. El alienista William Benham reclamaba ya en 1875 que los internos, desde su entrada al establecimiento, debían "luchar contra todas las circunstancias desfavorables del proceso de restablecimiento". Si bien esta crítica le valió su destitución como médico del asilo, ciertamente la Casa, no ofrecía las condiciones terapéuticas prometidas, ni tampoco garantizaba la ejecución del tratamiento moral, por problemas de toda índole, que consideraban hasta la ausencia de un reloj, símbolo de la rutina y de la disciplina que se buscaba implementar. Como decía Benham, sin "el conocimiento del tiempo" había sido casi imposible observar alguna regularidad en el recinto ${ }^{24}$.

La reflexión en torno al espacio y sus usos, así como la definición de sus paisajes, fueron parte importante de los debates que acompañaron la reforma de las instituciones asilares en Chile y en la región (Sacristán, 2008). Los viajes de los especialistas a Europa y Estados Unidos, desde la década del ochenta, para explorar distintas alternativas en el tratamiento de la locura, les permitieron conocer otras experiencias asilares y reflexionar sobre sus diferencias con el modelo chileno. A través de ellos se incorporaron en la discusión nacional nuevos ideales y paisajes que se construían desde sus percepciones y descripciones, publicadas y compartidas tanto en las revistas especializadas, como en la prensa. Estos contenidos funcionaron como materia prima de los nuevos proyectos asilares que se pensaban en el país, y a diferencia de las influencias que impulsaron el primer asilo, funcionarían como un suministro más directo para los arquitectos y autoridades que empujarían los proyectos locales.

Uno de los primeros elementos destacados por los médicos fue el emplazamiento y la extensión del territorio hospitalario. Para fines del siglo XIX la Casa de Orates de Santiago se había vuelto más estrecha y había perdido el carácter aislado que la caracterizó en sus inicios. En este escenario, los médicos pidieron reubicar a los asilos en espacios alejados. Algunos, como el doctor Aureliano Oyarzun, sugirieron ubicarlos en el campo, donde hay "mayor cantidad de terreno" 25. Aparte del sustrato económico de esta afirmación, la necesidad de terreno apelaba a solucionar el asunto del hacinamiento, y principalmente, a satisfacer el deseo de contar con espacios exteriores apropiados, en concordancia con la valorización del carácter curativo que adquirían los espacios externos. Como planteaba Oyarzún, el contar con terrenos adecuados, en tamaño y ubicación, permitiría que los enfermos tuvieran a su disposición jardines, lagos donde bogar y hasta bosques, elementos que influían "favorablemente en sus estraviadas facultades cerebrales" 26 .

La presencia de "jardines y parques", como indicaba Germán Greve, eran constitutivos de los asilos modernos $^{27}$. Enviado a Europa en 1893 para instruirse en los nuevos manicomios y en la electroterapia, Greve relató, al igual que lo haría un par de años después Manuel Segundo Beca, médico residente de la Casa de Orates, en su visita a los establecimientos ingleses, las bondades de la naturaleza en el cuidado de la locura. Beca destacó la decoración, las flores y la vista al exterior del clásico asilo de Bethlem en Londres y el extenso paño de terreno, "hábil y hermosamente cultivado", plantado de "árboles y bosquecillos y de bien acabados parques y jardines" del Sanatorio de Holloway, vecino a Windsor, un establecimiento de lujo y de carácter privado ${ }^{28}$. Estos elementos pese a que no se encontraban bien desarrollados en Chile, por lo estrecho de los terrenos hospitalarios y por su bajo presupuesto, tampoco eran inexistentes. Desde un inicio las áreas verdes fueron consideradas relevantes. La Casa de Orates de los Olivos, como proyectan sus mapas y fotografías, contó con amplios terrenos al aire libre, contabilizándose alrededor de diez patios y un parque, los que fueron presentados como signos del progreso moral de la institución. Estos lugares "bien plantados", con arboledas que entregaban sombra "contra los rayos del ardiente sol de verano", y con flores que crecían a su alrededor, daban "un aire de alegría y de frescura" que en palabras del alienista William Benham, producía "un buen efecto en los pacientes", en un contexto donde el mismo profesional reconocía que el desarrollo de actividades variadas, "un té en algún punto de la quinta con un poco de música o una 'zamacueca' u otros entretenimientos" ayudaban significativamente a la felicidad de los internos y a su curación ${ }^{29}$. Los médicos recomendaban paseos diarios, y también, excursiones fuera de sus murallas, como "paseos campestres", al menos "cinco o diez veces en el año" ${ }^{30}$. El propio reglamento de 1884 autorizaba a los internos del pensionado, a pasearse por la arboleda de la casa, acompañados de uno o más guardianes ${ }^{31}$. 
Los beneficios de la naturaleza también podían aprovecharse por medio de referencias indirectas y representaciones alegóricas, como una "sencilla decoración de los patios, pinturas divertidas e interesantes en las murallas i en los dormitorios; distribución de flores, pájaros, gatos, perros i otros animales de regalo, como también libros i diarios"32. La ornamentación de los Olivos fue simple, sin embargo, acogió espacios particulares, como el Teatro Grez, que desplegó un discurso visual que apoyó la significancia de la naturaleza dentro del proyecto moral. El salón, a diferencia del resto de la casa, ofreció un espacio decorado con grandes murales que incluían figuras de la literatura, como Homero, Dante y Goethe, musas mitológicas y amplias escenas campestres que en conjunto generaban un aire bucólico, y terapéutico, inédito para la institución, pero no para el proyecto asilar en general (Fuentes y Gallardo, 2014).

La naturaleza en su formato natural o reproducido buscaba rebajar el carácter institucional del asilo y fortalecer su perfil familiar. Como plantea Clare Hickman para el contexto victoriano, se consideraba que para que el tratamiento moral surtiera efecto, los asilos de locos debían asemejarse lo más posible a un hogar (Hickman, 2013). Los jardines y parques, huertas y terrenos, permitían que el establecimiento se alejara de la idea de prisión y se presentara como un lugar doméstico, amable y libre. En Chile este interés también fue parte del proyecto asilar, especialmente en las primeras décadas de funcionamiento. El mismo nombre de la institución "Casa de Orates" hacía eco de esa aspiración. Aplicado solamente a ciertas instituciones de beneficencia, que podrían haber compartido la necesidad de reflejar el ambiente familiar, la "Casa" buscaba proyectar y comunicar un cotidiano asilar que se asemejara lo más posible al espacio doméstico, que ofreciera sus beneficios, pero desprovisto de los problemas e inconvenientes que incidían sobre la locura. Como planteaba Greve, se debía "despojar" a estas instituciones "del carácter de lazaretos ú hospitales y por el contrario darles el de casas habitaciones confortables, cuya vida interior sea la más familiar posible"33. Esta tendencia se mostraba con más claridad en los espacios destinados a las elites. La cuidada escenografía del pensionado, con habitaciones amobladas, cortinajes y ornamentaciones, además de vincularse con los preceptos del tratamiento moral, cumplían funciones retóricas, reproducidas en las memorias de la institución y en la prensa, con el objeto de convencer a los miembros de las elites que la Casa de Orates era también un lugar adecuado para ellos.
Con el tiempo, el énfasis dado al espacio exterior, a parques, jardines y el terreno en general, se vinculó con la importancia que comenzaron a adquirir los nuevos sistemas "tan aplaudidos por distinguidos alienistas" de "puertas abiertas, non restraint y open door"34. Este protagonismo seguía un proceso de cambio que se expresaba en otras latitudes que, en palabras de Ricardo Campos, respondía a la dualidad que manifestaba el espacio asistencial, derivado de la ordenación de los enfermos mentales en base a nociones asociadas a la cronicidad y la peligrosidad social que diferenciaba los tipos de encierro según enfermos y condiciones mentales (Campos, 2001 y 2007). Pese a que estos principios de profilaxia mental no fueron mayormente aplicados en Chile, al menos para el periodo estudiado, incidieron en el desarrollo del Asilo de Temperancia de la Casa de Orates, y posteriormente, como se ha señalado, en la discusión de los cambios asilares. Así, cuando comenzó a barajarse la posibilidad de construir nuevos manicomios, su ubicación, las condiciones del terreno y sus usos, fueron temas recurrentes que respondieron a criterios económicos de valor y disponibilidad de la tierra, y al mismo tiempo, a los nuevos idearios médicos y profilácticos de la temprana psiquiatría. Si bien los siguientes establecimientos construidos en Chile no se inscribieron en su totalidad en el sistema de puertas abiertas, si comenzaron a incorporar gradualmente algunos de los elementos constitutivos del modelo, sea en discusiones preliminares o en su implementación.

El asilo de locos de Concepción fue uno de los primeros establecimientos estatales para el cuidado de la locura en inaugurarse tras el de Santiago ${ }^{35}$. Creado en septiembre de 1895 en un edificio perteneciente al Buen Pastor, se pensó para los enfermos del sur del país y los problemas que generaba su traslado a la capital en un país con más de 4.000 kilómetros de extensión (Sievers, 2012). La centralización del proyecto asilar había generado problemas en provincia. Los enfermos se acumulaban en recintos policiales $u$ hospitalarios en la espera de ser remitidos a Santiago, para embarcarse luego en un traslado penoso, en el que eran acompañados por un guardián, generalmente de policía, descrito como "torpe e ignorante a menudo", "vigoroso, de gran desarrollo muscular" elegido "para emplear la fuerza bruta, de la que se abusaba con frecuencia". Durante el viaje no contaban con asistencia médica, y en el caso de locuras furiosas se documentaban situaciones como la de un hombre de Valdivia que, poseedor de fortuna, se trasladó a Santiago acompañado de su médico, pero amarrado a una escalera ${ }^{36}$. 
El nuevo asilo de Concepción no solucionó los problemas, ni desahogó mayormente a la capital, sino más bien contribuyó a la institucionalización de una población que se encontraba en espacios periféricos. Por ello, las solicitudes de recursos por parte del Ministerio del Interior para mejorar la Casa de los Olivos y las conversaciones respecto a la necesidad de construir un nuevo establecimiento manicomial, actualizaron la discusión. Para juicio de algunos, el proyecto asilar no mejoraría solo con unas cuantas reparaciones, sino que requería repensar los espacios que se estaban destinando al cuidado de los locos, enfocándose no tanto en la construcción de espacios provisorios, como los galpones que algunos sugerían levantar en el ala sur de los Olivos, sino más bien en la edificación de establecimientos nuevos y apropiados ${ }^{37}$.

Como resultado, a inicios de la década de 1890 comenzó a diseñarse un nuevo asilo. En octubre de 1894 se aprobaron los planos y el presupuesto presentado por Carlos Barroilhet para levantar un manicomio en los terrenos de la chacra de la Providencia, situados al oriente de la capital, entre la Avenida Providencia y el Camino de Ñuñoa ${ }^{38}$. El lugar correspondía a un espacio alejado, "ubicado aguas arriba" de la ciudad, cercano a otros lugares de salud en construcción, como el Hospital del Salvador, también dirigido por Barroilhet. Los médicos celebraron la elección del lugar, al lado de los estanques de agua potable, "en medio de un paisaje encantador", cuyo terreno presentaba "condiciones hijiénicas inmejorables", sobre un suelo en declive y seco, con libre acceso de aire y luz, con agua pura en abundancia, "con un horizonte sin límites" enmarcado por la Cordillera de los Andes, "donde los alienados podrán admirar los paisajes que les rodean"39. El llamado Manicomio Nacional se presentaba como construcción extensa y soberbia, con 45 mil metros cuadrados construidos, pabellones aislados, la mayoría de dos pisos, diseminados en medio de jardines, totalmente diferente al espacio de la antigua loquería. A los edificios y áreas verdes, se sumaban las 10 hectáreas de la colonia agrícola, destinados primero a una población de 1.200 enfermos, y luego de 650, siguiendo los presupuestos psiquiátricos que permitían ordenar a los internos en criterios de peligrosidad y cronicidad ${ }^{40}$. Tras varios años de trabajo, el establecimiento se erigía como ejemplo del proceso de modernidad de las instituciones hospitalarias y en específico, asilares. Si bien, el ideal de una "casa" comenzaba a quedar atrás, seguía vigente el interés por la naturaleza y el espacio exterior. Sin embargo, pese al impulso, el proyecto no prospero. En la etapa final de construcción, el establecimiento fue ocupado por el batallón Escuela de Clases, que perpe- tuó su estadía en el establecimiento, lo que sumado a defectos sanitarios que atrasaron su uso por parte de los enajenados, finalmente terminaron por entregar en 1904 el edificio a los militares.

Las demandas por mejoras y la validación del espacio como base curativa hicieron eco en Concepción. Apenas dos años después de su fundación, las autoridades solicitaron el traslado de los enfermos a espacios más apropiados, como la Quinta Agrícola y el sector del Molino de Puchacai, lugares más higiénicos, por ubicación y altura. Se pensaba que estos espacios permitirían entregar un régimen de trabajo que siguiera "las colonias agrícolas para enajenados", que representaban para esos años, "una aspiración universal"41. El proyecto de un Manicomio Moderno se implementó finalmente en Concepción hacia 1915, con la aprobación de la construcción del Manicomio José Cardenio Avello en un terreno de 14 hectáreas en la esquina de Avenida Ignacio Collao con Irarrázaval, bajo la dirección del arquitecto francés Emilio Doyère, creador de varios edificios institucionales en el país. Este manicomio, al igual que el de Providencia, sería vendido al Ministerio de Guerra, traspaso que refleja las luchas internas y externas que debieron darse para la definición, implementación y luego conservación de las instituciones manicomiales en el país (León y Rojas, 2016).

El interés por modernizar el manicomio respondía a coyunturas internas, y también al surgimiento de nuevos paradigmas que invitaban a transformar la "Casa" en una "ciudad", con mayores espacios de circulación, interacción y autonomía para los internos, de acuerdo a la diversificación de su población, tanto enferma, como médica. Esta institución se comunicó y presentó en la prensa como "una verdadera ciudad en pequeño (...) una ciudad donde encuentran albergue tranquilo muchos de los infelices que han perdido la razón" ${ }^{42}$, y comenzó a pensarse desde los principios del Open Door. Este modelo buscaba una mayor libertad de los internos, puertas sin llaves, circulación no controlada, vigilancia sin coerción espacial, destinada a los convalecientes y a los enfermos tranquilos, pensionistas, y también a casos ya crónicos e incurables, como relataba el doctor Beca en 1898, en referencia al asilo de Binghamton ${ }^{43}$. Una década después, el informe que presentaba el doctor Joaquín Castro en el Congreso Internacional de Alienistas celebrado en Viena, informaba de la necesidad de aplicar este sistema en Chile $^{44}$. En este escenario de amplitud, donde el horizonte cobraba sentido, el exterior nuevamente fue sindicado como protagonista, ya no tanto en términos morales, sino económicos y productivos. 
La discusión en Chile sobre la conveniencia y viabilidad del modelo de Open Door surgió a fines del siglo XIX. Alcanzó mayor contingencia durante los procesos de definición de los nuevos manicomios. Para el caso del asilo Avello, en Concepción, la Junta de Beneficenci, en concordancia con las tensiones y diferencias entre el cuerpo administrativo y el cuerpo médico de estos establecimientos, no dio mayores indicaciones al arquitecto respecto del principio rector del establecimiento, indicándole que su deseo era que tuviese una capacidad para dos mil enfermos. Se eligió un modelo en forma de $x$, al considerarse que el modelo del Open Door no parecía ser recomendable para un país que contaba con solo dos manicomios y que no podía ofrecer las garantías de separación para locos tranquilos y furiosos. Sin embargo, la buena recepción del modelo de puertas abiertas, llevó a que se decidiera destinar la chacra anexa al Manicomio para que los locos tranquilos pudiesen trabajar y de esta forma, aplicar al menos, algunos de sus principios ${ }^{45}$.

El establecimiento de un proyecto de Open Door se concretó recién hacia fines de la década del veinte. El Plan General de Manicomios de Chile, aprobado por la Junta de Beneficencia en julio de 1929 con el objeto de asistir científicamente a los enfermos, dividió el país en tres zonas -norte, centro y sur- buscando descentralizar el servicio. En provincia, definió la construcción de espacios asistenciales, en su mayoría pabellones especiales asociados a hospitales provinciales, y en Santiago, indicó la construcción de un Hospital Psiquiátrico en la zona de la Casa de los Olivos, específicamente donde se ubicaba el Asilo de Temperancia. También estableció la creación de un Asilo Colonia en el fundo "El Peral" adquirido por el Estado en 1928, algunos años después de la compra de la Quinta Bella en Conchalí en 1923. El plan consideraba vender el Hospicio de Santiago, trasladar a los internos en estado de demencia a El Peral y a los inválidos a la Quinta Bella. Se autorizaba a la Junta Central de Beneficencia vender los terrenos de la Casa de Orates, conservando solo los espacios que se destinarían a Hospital Psiquiátrico, con el objeto de solventar la deuda del establecimiento y financiar el Plan de Manicomios ${ }^{46}$.

Este Plan General de Manicomios formalizó un proyecto que se había gestado ya hacia en 1925 con la aprobación para adquirir un predio agrícola para el desarrollo de un Open Door. Se buscaba una superficie con un mínimo de 1500 hectáreas, adquiriéndose finalmente el fundo Concepción del Peral para el establecimiento del Open Door destinado a cuatro mil enfermos. Junto con la compra del terreno, se debía planear el régimen a implementar, lo que requirió nuevamente el envío de una comisión compuesta por el Director de la Casa de Orates, Francisco Echenique, el médico subdirector, Gerónimo Letelier y el arquitecto de la Junta de Beneficencia Oscar Oyadenel, para que fuesen a estudiar modelos de puertas abiertas al extranjero, considerándose los establecimientos de Buenos Aires, Montevideo y Río de Janeiro, particularmente respecto a su funcionamiento, reglamento y edificación ${ }^{47}$. Así, nuevamente, sobre terrenos definidos como higiénicos, se perfiló un modelo asilar asociado a nuevos ideales psiquiátricos, que pese a su carácter renovado, continuaba confiando en el retiro y el encierro, asociado esta vez a uno de los principales criterios terapéuticos que avalaban la Open Door: el trabajo ${ }^{48}$.

\section{CONCLUSIONES}

El proyecto asilar, devenido en proyecto manicomial, se levantó sobre determinados espacios, cuya identificación y selección, reflejó parte de las dinámicas de apropiación y desarrollo de la terapia moral en Chile. En esos espacios, dibujó una serie de paisajes, forjados por sujetos, saberes y prácticas, entre varios otros elementos, que se proyectaron en el tiempo y que se posicionaron como elementos constitutivos de los asilos, fundantes y vinculantes. Estos paisajes cambiaron con el tiempo, de la mano de la transformación del alienismo decimonónico en la psiquiatría de inicios del siglo $X X$, pero mantuvieron vigente la idea de retiro y disciplina como base de curación, paralelo a la expansión de nuevas fuentes terapéuticas basadas en la terapia de shock y en la farmacología. Estos espacios fueron armados y reproducidos, compartidos y comunicados, como parte de las estrategias de la institución, y como aquel opuesto que permitía no solo definir la ciudad de los locos, sino también la de los cuerdos.

Los paisajes se abrieron a la comunidad a través de la prensa y las editoriales. Las crónicas describieron el interior cotidiano del asilo y en ocasiones sus falencias, mientras que las fotografías celebraron su mundo material: los volúmenes, los edificios, los patios y parques, como muestras del avance y de la concreción de los ideales terapéuticos, funcionando como soporte del proyecto. Los espacios de las casas y manicomios mostraron un paisaje compartido bajo aquellas ambiciones e ideales que forjaron el imaginario asilar. Así, espacios como los de la Casa de Orates, el Manicomio Avello y el Manicomio Nacional, se presentaron no solo como sostenes materiales y diferenciados de un modelo, sino como parte de un sistema integra- 
do de alegorías, estructuras y formas, que articuladas a determinadas narrativas y tipologías, calaron en el proyecto médico de la época y en la concepción de la locura, más allá de su concreción y diferenciación.

Las casas, asilos y manicomios se entendieron y discutieron desde su espacialidad. La problematización de las categorías patológicas usadas, las tasas de recuperación, la operatividad de otros medicamentos, entre varios otros temas, fueron determinantes del espacio y de sus cambios, pero también fueron subsumidos en el discurso público respecto a estas instituciones por el protagonismo que tuvo el espacio manicomial como espacio de realización e implementación concreto del ideal alienista y psiquiátrico. Así, los paisajes asilares, entendidos como parte de un discurso visual dinámico, surgidos del encuentro del espacio intervenido, con el espacio en sí, ofrecieron también un circuito de tránsito y construcción de sentido que superó las instituciones particulares, proyectándose como un panorama institucional que se extendió tanto en el espacio, como en el tiempo.

El proyecto asilar se describió, se representó y se ofreció, a través de sus paisajes, los que constituyeron por, sobre todo, medios de proyección de un ideario nacional y médico, en proceso de definición. En ellos destacaron panoramas interiores y exteriores, que hicieron converger civilización con naturaleza, y que aludieron a paisajes icónicos que buscaron comunicar el desarrollo material, científico y profesional logrado por el alienismo, las autoridades y los médicos, en las condiciones naturales y sociales dadas en Chile. Así, pese a que durante el siglo XX los manicomios seguirían cambiando significativamente, mantendrían un paisaje común, derivado de la dificultad de renovar y de la falta de recursos, que mantuvo el guiño decimonónico al régimen moral, paralelo al desarrollo e introducción de nuevos criterios patológicos y terapéuticos para comprender y tratar la locura.

Las condiciones espaciales hacen pensar en el poder psiquiátrico y en sus condiciones de implementación y acción. El desarrollo de los asilos de locos da cuenta de cambios significativos en el mundo médico y de la salud pública, pero también revela la distancia entre el hacer y el querer, en recintos que no lograron monopolizar las prácticas de cuidado y tratamiento de los enfermos mentales en un Chile en el que la locura continuó viviéndose, para fines del siglo XIX e inicios del XX, en las calles y en los espacios familiares.

\section{NOTAS}

1. Hasta el momento no ha sido posible consultar fichas clínicas de la Casa de Orates, salvo los partes de internación y egreso que se encuentran en los fondos de la Municipalidad e Intendencia de Santiago, en los Archivos Judiciales o en los fondos médicos del Museo de Medicina Enrique Laval. Si bien parte de los espacios físicos de las Casas de Orates se mantienen, existen pocos registros que aluden a ellos, por tanto se recurrió a fuentes diversas que entregaran pistas de su creación e implementación.

2. Dejean, Pedro (1856), Plano topográfico de la ciudad de Santiago, Chile, Biblioteca Nacional de Chile, Sala Medina.

3. Junta de Beneficencia (1901), Casa de Orates de Santiago. Actas de la Junta Directiva, 1854-1891. Documentos anteriores a la primera Acta: 1852-1854, Santiago, Imprenta Valparaíso de Federico T. Lathrop, p. V.

4. Beca, Manuel (1885), Sobre las enfermedades mentales en Chile. Recopilación i estudio de la Estadística de la Casa de Orates desde su fundación en 1852 hasta la fecha, Santiago, Imprenta Nacional, p. 5.

5. Elguero, José Ramón (1863), “Informe del Médico de la Casa de Locos", Memoria que el Ministro de Estado en el departamento del Interior presenta al Congreso Nacional de 1863, Santiago, Imprenta Nacional, p. 183.

6. Zilleruelo, Julio (1896), “Estudio sobre la hospitalización de la locura", Revista Chilena de Hijiene, Tomo III, pp. 77-114.

7. Los asilados incluían enfermos agudos y curables, crónicos incurables, degenerados conjénitos y degenerados secundarios y enajenados peligrosos y criminales considerados irresponsables por la justicia. MIM (1871), Santiago, Imprenta Nacional, p. 187 y Movimiento de la Casa de Orates (1899), 131 y Rojas Troncoso, H. (1929), "La asistencia de los alienados en Chile". En, Actas de la Primera Conferencia Latino Americana de Neurología, Psiquiatría y Medicina Legal. Tomo II, Buenos Aires, Imprenta de la Universidad, pp. 442-463.

8. Movimiento de la Casa de Orates de Santiago en el segundo semestre de 1895 (1896), Santiago, Imprenta Gutenberg, p. 6 y p. 27. El delirio alcohólico era un problema asociado principalmente a los hombres, correspondiendo a 95 de los 117 casos. Probablemente el bajo número de ingresados con esa etiqueta, se debía a que los casos de locura alcohólica eran transitorios y no requerían internación, en un momento además en que la patologización del consumo excesivo de alcohol recién comenzaba a ser debatida por los médicos.

9. Rojas Troncoso, H. (1929) "La asistencia de los alienados en Chile". En, Actas de la Primera Conferencia Latino Americana de Neurología, Psiquiatría y Medicina Legal, Tomo II, Buenos Aires, Imprenta de la Universidad, pp. 442-463, p. 445. 
10. El Diario Oficial, 23 de agosto de 1893.

11. Biblioteca Museo Enrique Laval (1898), Correspondencia de la Casa de Orates, p. 4 y p. 5.

12. "Casa de Locos. Lei sobre la materia. Santiago, 31 de julio de 1856". En Anguita, Ricardo (1912), Leyes promulgadas en Chile. Desde 1810 hasta el 1 de junio de 1913, Santiago, Imprenta, Litografia I Encuadernación Barcelona, tomo II, pp. 27-28 y "Reglamento para la Casa de Orates, Decreto de 19 de Diciembre de 1883". En (1889), Disposiciones Vigentes en Chile sobre Policía Sanitaria y Beneficencia Pública, Santiago, Roberto Miranda Editor, p. 128. Pese a que no fue muy frecuente, los propios internos podían solicitar su salida al juez letrado de la provincia del establecimiento.

13. Por ejemplo el de Murillo, Adolphe (1889), Hygiene et Assitance Publique au Chili, Paris, Exposition Universelle de Paris.

14.Junta de Beneficencia (1901), Casa de Orates de Santiago. Actas de la Junta Directiva, 1854-1891. Documentos anteriores a la primera Acta: 1852-1854, Santiago, Imprenta Valparaíso de Federico T. Lathrop, p. 209.

15. Sazié, Carlos (1881), "Influencia del trabajo y de las distracciones en el tratamiento de la enajenación mental", Revista Chile, tomo I, pp. 178-188.

16. El Diario llustrado, 22 de abril de 1902.

17. Greve, Germán (1895) “Corrrespondencia. Cartas sobre Manicomio", Revista Médica, tomo 23, feb-marzo, p. 71.

18. Benham, William (1875), Casa de Orates. Informe pasado al Ministerio del Interior, Santiago, Imprenta de la República, p. 24.

19. Fuentes, Alejandra y Gallardo, Ximena (2014), Locura y arte. Teatro Grez, Santiago, Salviat.

20. Biblioteca Museo Enrique Laval, Correspondencia de la Casa de Orates, 1898, 6.

21. Ugarte, Carlos (1901), Memoria sobre el laboratorio, Santiago, Imprenta Valparaíso de Federico T. Lathrop, p. 4.

22. Ulloa, Arturo (1905), Estudio estadísticio sobre mil doscientas autopsias i mui especialmente sobre la tuberculosis en Santiago, Santiago, Imprenta i Encuadernación Universitaria.

23. Ugarte, Carlos (1901), Memoria sobre el laboratorio, Santiago, Imprenta Valparaíso de Federico T. Lathrop, p. 13.

24. Benham, William T. (1875), Casa de Orates. Informe pasado al Ministerio del Interior, Santiago, Imprenta de la República, p. 12 y p. 30.

25. "22a. Sesión en 31 de agosto de 1893", Diario Oficial, 13 de septiembre de 1893, p. 1723.

26. “22a . Sesión en 31 de agosto de 1893”, Diario Oficial, 13 de septiembre de 1893, p. 1723.

27. Greve, Germán (1895), “Corrrespondencia. Cartas sobre Manicomio", Revista Médica, tomo 23, feb-marzo, p. 71

28. Beca, Manuel Segundo (marzo de 1898), “Ministerio del In- terior. Informe sobre visita a manicomios extranjeros", Diario Oficial, p. 542.

29. Benham, William T. (1875), Casa de Orates. Informe pasado al Ministerio del Interior, Santiago, Imprenta de la República, p. 9 y 22.

30. Marquez, Ernesto (1901), Consideraciones sobre las curaciones I mortalidad de la Casa de Orates de Santiago, Santiago, Imp. Centro Editorial La Prensa, p. 7.

31.Junta de Beneficencia (1901), Casa de Orates de Santiago. Actas de la Junta Directiva, 1854-1891. Documentos anteriores a la primera Acta: 1852-1854, Santiago, Imprenta Valparaíso de Federico T. Lathrop, p. 263.

32. Benham, William T. (1875), Casa de Orates. Informe pasado al Ministerio del Interior, Santiago, Imprenta de la República, p. 21.

33. Greve, Germán (1895), “Correspondencia. Cartas sobre Manicomio", Revista Médica, tomo 23, p. 71.

34. Valdivia, Samuel (1900), Breves consideraciones sobre el Servicio de Enajenados en Chile, Santiago, Imprenta Española, p. 16.

35. Durante el siglo XIX existieron otros espacios privados donde se acogió a los locos, por ejemplo, aquellos destinados a las mujeres y supervisados por monjas, como las del Buen Pastor y de la Preciosa Sangre.

36. Valdivia, Samuel (1900), Breves consideraciones sobre el Servicio de Enajenados en Chile, Santiago, Imprenta Española, p. 10 y 13.

37. “22a. Sesión en 31 de agosto de 1893”, Diario Oficial, 13 de septiembre de 1893, pp. 1722-1724.

38. “Correspondencia del Consejo”, Diario Oficial, 27 de octubre de 1894,

39.Zilleruelo, Julio (1896), “Estudio sobre la hospitalización de la locura", Revista Chilena de Hijiene, tomo III, pp. 77-114.

40. Valdivia, Samuel (1900), Breves consideraciones sobre el Servicio de Enajenados en Chile, Santiago, Imprenta Española, p. 23.

41. Diario Oficial, 10 de noviembre de 1896, p. 2374.

42. Diario Ilustrado, 22 de abril de 1902.

43. Diario Oficial, 14 de marzo de 1898, p. 637-638.

44. Diario Oficial, 21 de mayo de 1910, p. 5647.

45. Diario Oficial, 19 de noviembre 1915.

46. Diario Oficial, 17 de diciembre de 1925, p. 3097 y 7 de septiembre de 1929, pp. 4956-4957.

47. Diario Oficial, 21 de agosto de 1928.

48. Rojas Troncoso, H. (1929),"La asistencia de los alienados en Chile". En, Actas de la Primera Conferencia Latino Americana de Neurología, Psiquiatría y Medicina Legal, tomo II, Buenos Aires, Imprenta de la Universidad, 1929, p. 442-463, p. 447 y Fernando Delbés (1918), El trabajo en los alienados, Santiago, Sociedad Imprenta-Litografía Universo, p. 11. 


\section{BIBLIOGRAFÍA}

Araya, Claudia (2015), Profesionalización de la psiquiatría en Chile: saberes y prácticas, 1826-1949, Tesis no publicada para acceder al grado de Doctor en Historia, Pontificia Universidad Católica de Chile.

Campos, Ricardo (2001), "De la higiene del aislamiento a la higiene de la libertad. La reforma de la institución manicomial en Francia (1860-1940)", Frenia, I, pp. 37-64.

Campos, Ricardo (2007), “Crimen y locura. La patologización del crimen en la España de la restauración", Norba. Revista de Historia, 20, pp. 85-105

Camus, Pablo (1993), “Filantropía, medicina y locura: la Casa de Orates de Santiago, 1852-1894", Historia, 27, pp. 89-140.

Castillo, Simón (2014), El Río Mapocho y sus riberas. Espacio público e intervención urbana en Santiago de Chile (18851918), Santiago, Ediciones Universidad Alberto Hurtado.

Correa, María José (2009), “Violencias ejercidas en los cuerpos enajenados: encierro terapéutico y privación de derechos civiles. Chile central (1850-1870)", Nuevo Mundo Mundos Nuevos, Debates, 9, pp. 1-19.

Correa, María José (2013), Historias de Locura e Incapacidad. Santiago y Valparaíso (1857-1900), Santiago, Acto Editores.

Cosgrove, Denis (2002), “Observando la naturaleza: el paisaje y el sentido europeo de la vista”, Boletín de la A.G.E.N., 34, pp. 63-89.

Edginton, Barry (2003), "The Design of Moral Architecture at the York Retreat", Journal of Design History, 16 (2), pp. 103-117.

Fernández, Marcos (2009), "Fuera de sí: cuerpo, ebriedad y conciencia en Chile. 1870-1940". En: Góngora, Alvaro; Sagredo, Rafael (dirs.), Fragmentos para una historia del cuerpo en Chile, Santiago, Taurus, pp. 285-327.

Foucault, Michel y Miskowiec, Jay (1986), "Of Other Spaces", Diacritics, 16 (1), pp. 22-27.

Fuentes, Alejandra y Gallardo, Ximena (2014), Locura y arte. Teatro Grez, Santiago, Salviat.

Goffman, Erving (1961), Asylum, Nueva York, Doubleday Anchor Books.

Goldberg, Ann (2001), Sex, Religion, and the Making of Modern Madness. The Eberbach Asylum and German Society, 18151840, Oxford, Oxford University Press.

Grob, Gerald (1973), Mental Institutions in América: Social Policy to 1875 , Londres, Collier-Macmillan.

Hickman, Clare (2005), "The Picturesque at Brislington House, Bristol: The Role of Landscape in Relation to the Treatment of Mental Illness in the Early Nineteenth-Century Asylum", Garden History, 33 (1), pp. 47-60.

Hickman, Clare (2013), Therapeutic Landscapes. A History of English Hospitals Gardens since 1800, Manchester, Manchester University Press.
Huertas, Rafael (2008), Los laboratorios de la norma: medicina y regulación social en el estado liberal, Barcelona, Octaedro-CSIC.

León, Marco y Rojas, Mauricio (2016), “Entre la defensa y la intervención social: Estado, ciudadanía y pobreza en la ciudad de Concepción (Chile, 1890-1930), Revista de Indias, LXXVI (268), pp. 821-850.

Leyton, César (2005), La ciudad médica-industrial: melancólico, delirante y furioso. El Psiquiátrico de Santiago de Chile, 1825-1930, Tesis no publicada para acceder al grado de Magíster en Historia, Universidad de Chile.

MacKinnon, Dolly (2009), “Divine Service, Music, Sport, and Recreation as Medicinal in Australian Asylum 1860s-1945", Health and History, 11 (1), pp. 128-148.

Mitchell, William John Thomas (2002), Landscape and Power, Chicago, University of Chicago Press.

Osorio, Carlos (2016), "Historia de los terrenos de la Casa de Orates de Santiago de Chile", Revista Médica de Chile, 144, pp. 388-393.

Pita, Valeria Silvina (2012), La casa de las locas. Una historia social del Hospital de Mujeres Dementes. Buenos Aires, 18521890, Rosario, Prohistoria.

Ponce de León, Macarena (2011), Gobernar la pobreza. Prácticas de caridad y beneficencia en la ciudad de Santiago, 1830-1890, Santiago, Editorial Universitaria, DIBAM.

Rothman, David J. (1971), The Discovery of the Asylum: Social Order and Disorder in the New Republic, Boston, Little, Brown \& Co.

Ruperthuz, Mariano y Sánchez, Marcelo (2015), “Entre la degeneración y el psicoanálisis: una pericia médico legal chilena en 1915", Revista Historia y Justicia, 4, pp. 138-168.

Sacristán, Cristina (2008), "Para integrar a la Nación. Terapéutica deportiva y artística en el Manicomio La Castañeda en un momento de reconstrucción nacional, 1920-1940". En Agostini, Claudia (coord.), Curar, sanar y educar: enfermedad y sociedad en México, siglos XIX y XX, México, UNAM, pp. 99-123.

Sacristán, Cristina (2009), "La locura se topa con el manicomio", Cuicuilco, 16 (45), pp. 163-189.

Sánchez, Marcelo (2014), "La teoría de la degeneración en Chile (1892-1915)", Historia, 47 (2), pp. 375-400.

Sievers, Alexander (2012), "Beneficencia, asilos para enajenados y su relación con la transformación del Estado en Concepción, durante las primeras décadas del siglo XX", Tiempo y Espacio, 28, pp. 151-182.

Torres, Luis Alberto (2014), "Tratamiento moral y condiciones de encierro en la Casa de Orates: los límites del proceso de medicalización en Santiago, 1852-1891", Revista de Historia Social y de las Mentalidades, 18 (1), pp. 85-108. 